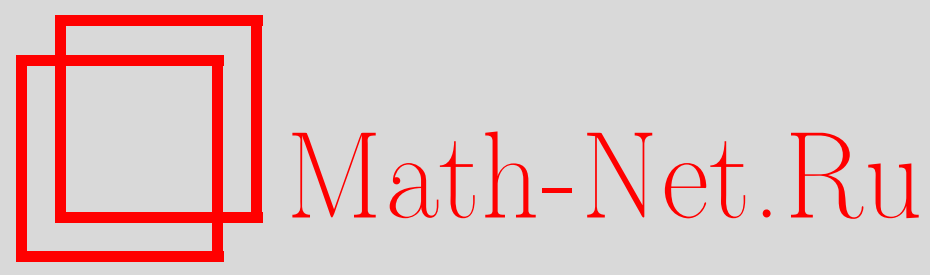

В. П. Маслов, Об одном новом вариационном принципе для фермионов, Матем. заметки, 1997, том 62, выпуск 4, 633634

DOI: https://doi.org/10.4213/mzm1648

Использование Общероссийского математического портала Math-Net.Ru подразумевает, что вы прочитали и согласны с пользовательским соглашением http://www . mathnet.ru/rus/agreement

Параметры загрузки:

IP : 3.89 .197 .203

26 апреля 2023 г., 03:18:23

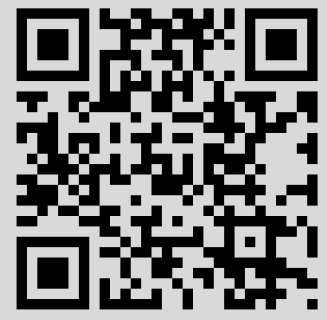




\section{ОБ ОДНОМ НОВОМ ВАРИАЦИОННОМ ПРИНЦИПЕ ДЛЯ ФЕРМИОНОВ}

\section{В. П. Маслов}

Вариационньй принцип Хартри-Фока описывает независимые ("холостые") электроны; обобщающий его вариационньй принцип Боголюбова описьвает как "холостые" электроны, так и “куперовские пары" - “женатые" электроны. Ситуация, когда все электроны спарены (вблизи уровня Ферми), отвечает определенному состоянию (сверхтекучести): если заданы $2 N$ частиц $x_{1}, x_{2}, \ldots, x_{2 N}, x_{i} \in \mathbb{R}^{3}$, то спаренными являются, например, $\left(x_{1}, x_{2}\right),\left(x_{3}, x_{4}\right), \ldots,\left(x_{2 N-1}, x_{2 N}\right)$. Антисимметрическая структура дает сумму всевозможных комбинаций "женатых" пар, например, $\left(x_{1}, x_{3}\right),\left(x_{2}, x_{4}\right), \ldots$. Каж дый член этой суммы будет содержать определенные пары ( “моногамия”).

Оказьвается, что, вообще говоря, возможна ситуация, когда все фермионы спарены между собой, образуя одновременно все комбинации пар, именно,

$$
\left(x_{1}, x_{2}\right),\left(x_{1}, x_{3}\right), \ldots,\left(x_{1}, x_{N}\right),\left(x_{2}, x_{3}\right),\left(x_{2}, x_{4}\right), \ldots,\left(x_{2}, x_{N}\right), \ldots,\left(x_{N-1}, x_{N}\right),
$$

образуя “клубок", в котором каждый “женат" сразу на всех остальных ("гарем"), и который труднее "разорвать", чем разбить куперовскую пару. Возможно, такая структура отвечает ядерным силам.

Рассмотрим уравнение Шрёдингера для $N$ тождественных частищ:

$$
\begin{aligned}
\widehat{\mathscr{H}}_{N} \Psi & \equiv\left(\sum_{i=1}^{N}\left(-\frac{\hbar^{2}}{2 m} \Delta_{x_{i}}+U\left(x_{i}\right)\right)+\varepsilon \sum_{1 \leqslant i<j \leqslant N} V\left(x_{i}-x_{j}\right)\right) \Psi\left(x_{1}, \ldots, x_{N}\right) \\
& =E \Psi\left(x_{1}, \ldots, x_{N}\right), \quad x_{i} \in \mathbb{R}^{3} .
\end{aligned}
$$

Здесь $U(x)$ - внешний потенциал,

$$
\varepsilon V\left(x_{i}-x_{j}\right)=V_{0} v\left(\frac{\left|x_{i}-x_{j}\right|}{a}\right)
$$

- потенциал взаимодействия между частицами, $m$ - масса частищы.

Будем искать минимум функционала

$$
I[\Psi]=\int d x_{1} \cdots d x_{N} \Psi^{*}\left(\widehat{\mathscr{H}}_{N}-E\right) \Psi
$$

в классе функций

$$
\Psi\left(x_{1}, \ldots, x_{N}\right)=\prod_{1 \leqslant i<j \leqslant N} \Phi\left(x_{i}, x_{j}\right), \quad \Phi(x, y)=-\Phi(y, x) .
$$

Тогда получаем

$$
\begin{aligned}
& \int d x_{3} \cdots d x_{N}\left(\prod_{i=3}^{N} \Phi^{*}\left(x_{1}, x_{i}\right) \Phi^{*}\left(x_{2}, x_{i}\right)\right) \cdot \prod_{3 \leqslant j<k \leqslant N} \Phi^{*}\left(x_{j}, x_{k}\right) \\
& \times\left(\sum_{l=1}^{N}\left(-\frac{\hbar^{2}}{2 m} \Delta_{x_{l}}+U\left(x_{l}\right)\right)+\varepsilon \sum_{1 \leqslant l<n \leqslant N} V\left(x_{l}-x_{n}\right)-E\right) \cdot \prod_{1 \leqslant p<q \leqslant N} \Phi\left(x_{p}, x_{q}\right)=0 . \\
& 1997
\end{aligned}
$$


Вариационньй принцип Хартри-Фока дает точньй ответ в случае, когда потенциал взаимодействия равен 0; вариационный принцип Боголюбова дает точньй ответ для модели БКШ. Приведенньй вариационньй метод совпадает с главным членом квазиклассической асимптотики для основного состояния гладкого положительного потенциала взаимодействия (притяжения):

$$
V(x)>0 \quad \text { при } x \neq 0, \quad V(0)=0, \quad U(x) \equiv 0 \text {. }
$$

Действительно, в последнем случае асимптотика дается каноническим оператором, отвечаюшим ростку в точке покоя $p=0, x=0$ задачи

$$
-\frac{\hbar^{2}}{2 m} \Delta \Phi_{1}+V(x) \Phi_{1}=\lambda_{1} \Phi_{1}
$$

где $\lambda_{1}$ - первое возбужденное состояние (с единичным оператором рождения),

$$
\Phi_{1}=\Phi_{1}^{0}(x, \hbar)+O\left(\hbar^{2}\right), \quad \Psi_{0}\left(x_{1}, \ldots, x_{N}\right)=\frac{1}{N !}\left(\prod_{i<j} \Phi_{1}^{0}\left(x_{i}-x_{j}, \hbar\right)+O\left(\hbar^{2}\right)\right) .
$$

Энергия основного состояния равна

$$
E_{0}=V_{0} \cdot \frac{\hbar}{a \sqrt{m V_{0}}} \cdot \sqrt{N \frac{d^{2} v}{d r^{2}}(0)} \cdot \frac{N^{2}-1}{2}+V_{0} \cdot O\left(\frac{\hbar^{2}}{m a^{2} V_{0}}\right)
$$

энергия первого возбужденного состояния равна

$$
E_{1}=E_{0}+2 V_{0} \cdot \frac{\hbar}{a \sqrt{m V_{0}}} \cdot \sqrt{N \frac{d^{2} v}{d r^{2}}(0)}+V_{0} \cdot O\left(\frac{\hbar^{2}}{m a^{2} V_{0}}\right) .
$$

Таким образом, при $N \rightarrow \infty$ энергия, требуемая для возбуждения, пропорциональна $\sqrt{N}$. Если $v(|x-y|)$ стремится к 0 при $|x-y| \rightarrow 0$ достаточно быстро, то матричньй элемент перехода (для произвольного $\hbar$-псевдодифференциального оператора с гладким убывающим символом) между основным состоянием и всеми “холостьми” фермионами экспоненциально мал при $\hbar \rightarrow 0, N \rightarrow \infty$.

Если уравнения Хартри-Фока и Боголюбова-БКШ определяют асимптотику при $N \rightarrow \infty$, то предложенньй вариационньй метод отвечает задачам, в которых $\hbar \rightarrow 0$, $N \rightarrow \infty$ при некоторых соотношениях между $\hbar$ и $N$.

Автор приносит благодарность аспирантам Г. Ковалю и А. Рууге за помощь в вычислениях. 